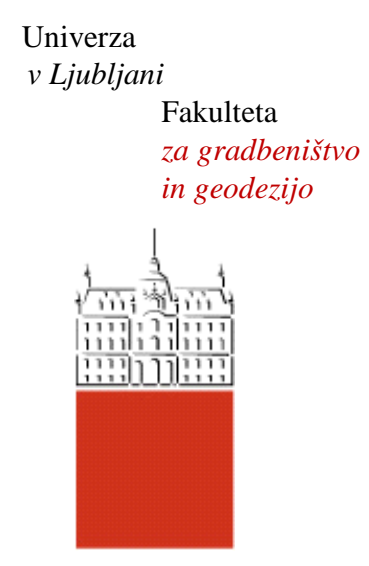

Jamova 2

1000 Ljubljana, Slovenija http://www3.fgg.uni-lj.si//

DRUGG - Digitalni repozitorij UL FGG http://drugg.fgg.uni-lj.si/

Ta članek je avtorjeva zadnja recenzirana različica, kot je bila sprejeta po opravljeni recenziji.

Prosimo, da se pri navajanju sklicujte na bibliografske podatke, kot je navedeno:
University
of Ljubljana

Faculty of Civil and Geodetic Engineering

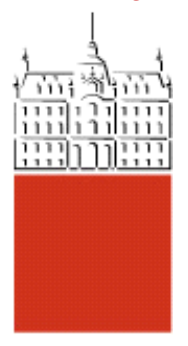

Jamova 2

SI - 1000 Ljubljana, Slovenia

http://www3.fgg.uni-lj.si/

DRUGG - The Digital Repository http://drugg.fgg.uni-lj.si/

This version of the article is author's manuscript as accepted for publishing after the review process.

When citing, please refer to the publisher's bibliographic information as follows:

Dujc, J., Brank, B., Ibrahimbegović, A., Brancherie, D. 2010. An embedded crack model for failure analysis of concrete solids. Computers and Concrete 7, 4: 331-346. 


\title{
An embedded crack model for failure analysis of concrete solids
}

\author{
Jaka Dujc ${ }^{1}$, Boštjan Brank ${ }^{1, *}$, \\ Adnan Ibrahimbegovic ${ }^{2}$ and Delphine Brancherie ${ }^{3}$ \\ ${ }^{1}$ University of Ljubljana, Faculty of Civil and Geodetic Engineering, Slovenia \\ ${ }^{2}$ École Normale Supérieure de Cachan, France \\ ${ }^{3}$ Université de Technologie de Compiègne, France \\ *corresponding author; e-mail: bbrank@fgg.uni-lj.si
}

January 27,2010

\begin{abstract}
We present a quadrilateral finite element with an embedded crack that can be used to model tensile fracture in two-dimensional concrete solids and the crack growth. The element has kinematics that can represent linear jumps in both normal and tangential displacements along the crack line. The cohesive law in the crack is based on rigid-plasticity with softening. The required material data for the concrete failure analysis are the constants of isotropic elasticity and the mode $I$ softening curve. The results of two well known tests are presented in order to illustrate very satisfying performance of the presented approach to simulate failure of concrete solids.
\end{abstract}

\section{Introduction}

Numerical finite element simulations of solids, that consider material failure, are not reliable if the finite element formulations are based on the classical continuum mechanics. Mesh-dependent solutions are obtained. Different approaches have been proposed to overcome this problem: non-local continuum, higher order gradient plasticity, viscoplastic regularization, Cosserat continuum, and some others (see e.g. [3] for a recent review on the topic). Those remedies are nowadays often replaced by a modification of the classical continuum model, which allows for discontinuity in displacements, e.g. [11]. We talk about the embedded discontinuity model. An embedded discontinuity finite element model can provide an adequate inelastic dissipation in the discontinuity, regardless of the chosen finite element mesh. This ensures mesh-independent solutions of simulations that include material failure. For a review on different approaches towards derivation of the embedded discontinuity model we refer to [11]. 
In this work we present a finite element formulation of the embedded discontinuity model that is based on the quadrilateral two-dimensional finite element. The majority of works related to the modeling of failure in two-dimensional solids by the embedded strong discontinuity finite element, e.g. [10], [6], [12], [2], [11], are based on the simple constant strain triangle. The reason for this choice might be that more complex elements, like the quadrilateral element, are much more difficult to design to be free of locking when the embedded discontinuity is added to the finite element formulation, e.g. [5], [9]. Quadrilateral finite element with embedded discontinuity, which is presented in this work, does not show any locking problems. This is mostly due to its enriched kinematics that can model linear jumps in displacements along the discontinuity line in both normal and tangential directions.

The derived quadrilateral finite element with embedded discontinuity is used for description of tensile fracture process in the two-dimensional plain concrete solids and to model the crack growth. Relatively simple constitutive equations are used for that purpose: the plane strain/plane stress linear elasticity for the bulk concrete material and the rigid-plasticity with softening for the cohesive law in the crack. The required material data are the constants of isotropic elasticity and the mode $I$ softening curve. This kind of cohesive law is fine for the case of monotonically increasing loading when the crack width increases monotonically with the load. However, when the loading is cyclic or the crack opens and closes during the loading process, a more demanding cohesive model would be needed, e.g. the one based on the modeling of damage, e.g. [12], [18].

The two-dimensional embedded crack formulation for plain concrete presented below can be extended to three-dimensions and to the reinforced concrete members. For the embedded crack modeling of reinforced concrete members we refer to e.g. [15], [19] and for the three-dimensional embedded crack analysis of concrete members we refer to [16], [17].

\section{Quadrilateral finite element with embedded crack line}

In this section we briefly present a quadrilateral two-dimensional plane stress/plane strain finite element with embedded crack line. The element assumes elastic material response up to the crack initiation and rigid-plastic softening response along the crack line during the crack opening.

\section{$2.1 \quad$ Kinematics}

Let us consider a quadrilateral finite element occupying domain $\Omega^{e}$ in $\mathbb{R}^{2}$. The element may be divided by the crack line $\Gamma^{e}$ into two subdomains, $\Omega^{e+}$ and $\Omega^{e-}$ $\left(\Omega^{e}=\Omega^{e+} \cup \Omega^{e-}\right)$. Element's geometry is defined by the bi-linear mapping $\boldsymbol{\xi}$ 
$\mapsto \boldsymbol{x}\left(\boldsymbol{\xi} \in[-1,1] \times[-1,1] ; \boldsymbol{x} \in \Omega^{e}\right)$ with

$$
\left.\boldsymbol{x}(\boldsymbol{\xi})\right|_{\Omega^{e}}=\sum_{a=1}^{4} N_{a}(\boldsymbol{\xi}) \boldsymbol{x}_{a}, \quad \boldsymbol{x}_{a}=\left[x_{a}, y_{a}\right]^{T}, \quad \boldsymbol{\xi}=[\xi, \eta]^{T},
$$

where $\boldsymbol{x}_{a}$ are coordinates of the finite element node $a$ and

$$
N_{a}(\boldsymbol{\xi})=\frac{1}{4}\left(1+\xi_{a} \xi\right)\left(1+\eta_{a} \eta\right), \quad \begin{array}{lllll}
a & 1 & 2 & 3 & 4 \\
\hline \xi_{a} & -1 & 1 & 1 & -1 \\
\hline \eta_{a} & -1 & -1 & 1 & 1
\end{array} .
$$

To model the crack opening, the parameters $\alpha_{n 0}, \alpha_{n 1}, \alpha_{m 0}$ and $\alpha_{m 1}$ are introduced along the crack line $\Gamma^{e}$. The mid-point of the crack line is denoted by $\boldsymbol{x}_{\Gamma}$. We assume the element displacement field as:

$$
\begin{aligned}
& {\left[u_{x}, u_{y}\right]^{T}=\boldsymbol{u}\left(\boldsymbol{\xi}, \Gamma^{e}\right)=\underbrace{\sum_{a=1}^{4} N_{a}(\boldsymbol{\xi}) \boldsymbol{d}_{a}}_{\boldsymbol{u}_{d}}+} \\
& +\underbrace{\boldsymbol{M}_{n 0}\left(\boldsymbol{\xi}, \Gamma^{e}\right) \alpha_{n 0}+\boldsymbol{M}_{n 1}\left(\boldsymbol{\xi}, \Gamma^{e}\right) \alpha_{n 1}+\boldsymbol{M}_{m 0}\left(\boldsymbol{\xi}, \Gamma^{e}\right) \alpha_{m 0}+\boldsymbol{M}_{m 1}\left(\boldsymbol{\xi}, \Gamma^{e}\right) \alpha_{m 1}}_{\boldsymbol{u}_{\alpha}} .(3)
\end{aligned}
$$

Here, $\boldsymbol{d}_{a}=\left[u_{x a}, u_{y a}\right]^{T}$ are the nodal values of displacements, and $\boldsymbol{u}_{\alpha}$ are displacements due to the crack opening. Interpolation matrices $\boldsymbol{M}_{n 0}\left(\boldsymbol{\xi}, \Gamma^{e}\right)$, $\boldsymbol{M}_{n 1}\left(\boldsymbol{\xi}, \Gamma^{e}\right), \boldsymbol{M}_{m 0}\left(\boldsymbol{\xi}, \Gamma^{e}\right)$ and $\boldsymbol{M}_{m 1}\left(\boldsymbol{\xi}, \Gamma^{e}\right)$ are related to the introduced crackopening parameters. With four introduced crack-opening parameters we are in position to model the following four independent modes of element separation along $\Gamma^{e}$ (see Figure 1):

1. " $n 0 "$ - constant opening in the direction of the crack normal $\mathbf{n}=\left[n_{x}, n_{y}\right]^{T}$,

2. " $n 1 "$ - linear opening in the direction of the crack normal $\mathbf{n}$ is linear; this mode corresponds to the rotation of $\Omega^{e+}$ around $\boldsymbol{x}_{\Gamma}$,

3. " $m 0 "$ - constant opening in the direction of the crack line, which is defined by $\mathbf{m}=\left[m_{x}, m_{y}\right]^{T}$,

4. " $m 1 "$ - linear opening in the direction of the crack line $\mathbf{m}$; this mode corresponds to the stretching of $\Omega^{e+}$ in the direction of $\pm \mathbf{m}$ with zero stretching at $\boldsymbol{x}_{\Gamma}$.

Displacements of $\Omega^{e-}$ and $\Omega^{e+}$ for a particular separation mode are equal to, see (3),

$\boldsymbol{u}_{\text {mode }}=\boldsymbol{u}_{d, \text { mode }}+\boldsymbol{u}_{\alpha, \text { mode }}, \quad \boldsymbol{u}_{\alpha, \text { mode }}=\boldsymbol{M}_{\text {mode }} \alpha_{\text {mode }}, \quad$ mode $\in[n 0, n 1, m 0, m 1]$.

From (4) it follows

$$
\boldsymbol{M}_{\text {mode }}=\frac{\boldsymbol{u}_{\text {mode }}-\boldsymbol{u}_{d, \text { mode }}}{\alpha_{\text {mode }}} .
$$


By examining Figure 1 we can determine $\boldsymbol{u}_{\text {mode }}$ and $\boldsymbol{u}_{\alpha, \text { mode }}$. By using (5), we can further derive the interpolation matrices $\boldsymbol{M}_{\text {mode }}$ (see [1] for details). Their forms are:

1. for constant normal separation mode " $n 0 "$ :

$$
\boldsymbol{M}_{n 0}=\left(H_{\Gamma}(\boldsymbol{x})-\sum_{a \in \Omega^{e+}} N_{a}\right) \boldsymbol{n}, \quad H_{\Gamma}(\boldsymbol{x})=\left\{\begin{array}{cc}
1 & \text { for } \boldsymbol{x} \in \Omega^{e+} \\
0 & \text { otherwise }
\end{array},\right.
$$

2. for linear normal separation mode " $n 1 "$ :

$$
\boldsymbol{M}_{n 1}=H_{\Gamma}(\boldsymbol{x})\left[\begin{array}{cc}
0 & 1 \\
-1 & 0
\end{array}\right] \overline{\boldsymbol{x}}-\sum_{a \in \Omega^{e+}} N_{a}\left[\begin{array}{cc}
0 & 1 \\
-1 & 0
\end{array}\right] \overline{\boldsymbol{x}}_{a}, \quad \overline{\boldsymbol{x}}=\boldsymbol{x}-\boldsymbol{x}_{\Gamma}
$$

3. for constant tangential separation mode " $m 0$ ":

$$
\boldsymbol{M}_{m 0}=\left(H_{\Gamma}(\boldsymbol{x})-\sum_{a \in \Omega^{e+}} N_{a}\right) \boldsymbol{m}
$$

4. for linear tangential separation mode " $m 1 "$ :

$$
\boldsymbol{M}_{m 1}=\left(H_{\Gamma}(\boldsymbol{x}) \boldsymbol{m} \cdot \boldsymbol{x}-\sum_{a \in \Omega^{e+}} N_{a} \boldsymbol{m} \cdot \boldsymbol{x}_{a}\right) \boldsymbol{m} .
$$

The vector of small strains at $\boldsymbol{x} \in \Omega^{e}$

$$
\boldsymbol{\epsilon}=\left[\frac{\partial u_{x}}{\partial x}, \frac{\partial u_{y}}{\partial y}, \frac{\partial u_{x}}{\partial y}+\frac{\partial u_{y}}{\partial x}\right]^{T},
$$

is obtained from (3) and (6)-(9) (see [1] for details) and can be written as

$$
\boldsymbol{\epsilon}=\sum_{a=1}^{4} \boldsymbol{B}_{a} \boldsymbol{d}_{a}+\boldsymbol{G}_{n 0} \alpha_{n 0}+\boldsymbol{G}_{n 1} \alpha_{n 1}+\boldsymbol{G}_{m 0} \alpha_{m 0}+\boldsymbol{G}_{m 1} \alpha_{m 1}
$$


where

$$
\begin{aligned}
& \boldsymbol{B}_{a}=\left[\begin{array}{cc}
\frac{\partial N_{a}}{\partial x} & 0 \\
0 & \frac{\partial N_{a}}{\partial y} \\
\frac{\partial N_{a}}{\partial y} & \frac{\partial N_{a}}{\partial x}
\end{array}\right], \boldsymbol{B}_{n}=\left[\begin{array}{cc}
n_{x} & 0 \\
0 & n_{y} \\
n_{y} & n_{x}
\end{array}\right], \boldsymbol{B}_{m}=\left[\begin{array}{cc}
m_{x} & 0 \\
0 & m_{y} \\
m_{y} & m_{x}
\end{array}\right] \\
& \boldsymbol{G}_{n 0}=\underbrace{-\sum_{a \in \Omega^{e+}} \boldsymbol{B}_{a} \boldsymbol{n}}_{\overline{\boldsymbol{G}}_{n 0}}+\underbrace{\delta_{\Gamma} \boldsymbol{B}_{n} \boldsymbol{n}}_{\overline{\overline{\boldsymbol{G}}}_{n 0}}, \quad \delta_{\Gamma}(\boldsymbol{x})=\left\{\begin{array}{cc}
\infty & \text { for } \boldsymbol{x} \in \Gamma^{e} \\
0 & \text { otherwise }
\end{array},\right. \\
& \boldsymbol{G}_{n 1}=\underbrace{-\sum_{a \in \Omega^{e+}} \boldsymbol{B}_{a}\left[\begin{array}{cc}
0 & 1 \\
-1 & 0
\end{array}\right] \boldsymbol{x}_{a}}_{\overline{\boldsymbol{G}}_{n 1}}+\underbrace{\delta_{\Gamma} \boldsymbol{B}_{n} \boldsymbol{n} \xi_{\Gamma}}_{\overline{\overline{\boldsymbol{G}}}_{n 1}}, \\
& \boldsymbol{G}_{m 0}=\underbrace{-\sum_{a \in \Omega^{e+}} \boldsymbol{B}_{a} \boldsymbol{m}}_{\overline{\boldsymbol{G}}_{m 0}}+\underbrace{\delta_{\Gamma} \boldsymbol{B}_{n} \boldsymbol{m}}_{\overline{\overline{\boldsymbol{G}}}_{m 0}} \\
& \boldsymbol{G}_{m 1}=\underbrace{H_{\Gamma} \boldsymbol{B}_{m} \boldsymbol{m}-\sum_{a \in \Omega^{e+}} \boldsymbol{B}_{a}\left(\boldsymbol{m} \cdot \overline{\boldsymbol{x}}_{a}\right) \boldsymbol{m}}_{\overline{\boldsymbol{G}}_{m 1}}+\underbrace{\delta_{\Gamma} \boldsymbol{B}_{n} \boldsymbol{m} \xi_{\Gamma}}_{\overline{\overline{\boldsymbol{G}}}_{m 1}} .
\end{aligned}
$$

In (14) and (16) we denoted by $\xi_{\Gamma} \in\left[-l_{\Gamma} / 2, l_{\Gamma} / 2\right]$ a coordinate along $\Gamma^{e}$, which has value 0 at $\boldsymbol{x}_{\Gamma}$ and is positive in the direction of $\boldsymbol{m}$. The $l_{\Gamma}$ represents the length of the crack. According to (13)-(16) the strains (11) can be divided into a regular part $\overline{\boldsymbol{\epsilon}}$ and a singular part $\overline{\overline{\boldsymbol{\epsilon}}}$ as

$$
\begin{aligned}
\boldsymbol{\epsilon} & =\overline{\boldsymbol{\epsilon}}+\overline{\overline{\boldsymbol{\epsilon}}} \\
\overline{\boldsymbol{\epsilon}} & =\sum_{a=1}^{4} \boldsymbol{B}_{a} \boldsymbol{d}_{a}+\overline{\boldsymbol{G}}_{n 0} \alpha_{n 0}+\overline{\boldsymbol{G}}_{n 1} \alpha_{n 1}+\overline{\boldsymbol{G}}_{m 0} \alpha_{m 0}+\overline{\boldsymbol{G}}_{m 1} \alpha_{m 1} \\
\overline{\overline{\boldsymbol{\epsilon}}} & =\overline{\overline{\boldsymbol{G}}}_{n 0} \alpha_{n 0}+\overline{\overline{\mathbf{G}}}_{n 1} \alpha_{n 1}+\overline{\overline{\boldsymbol{G}}}_{m 0} \alpha_{m 0}+\overline{\overline{\mathbf{G}}}_{m 1} \alpha_{m 1}
\end{aligned}
$$

where the singular part is just a particular representation of the localized deformation at the crack.

The kinematic enrichment of the standard quadrilateral element, which leads to the strains (18)-(19), is viewed in what follows in the manner of the incompatible modes, e.g. [4].

\subsection{Constitutive equations}

We assume that the behavior of the concrete can be described by the plane stress/plane strain elasticity

$$
\boldsymbol{\sigma}=\boldsymbol{C} \boldsymbol{\epsilon}, \quad \boldsymbol{\sigma}=\left[\sigma_{x}, \sigma_{y}, \sigma_{x y}\right]^{T}
$$

up to the crack appearance. Here, $\boldsymbol{\sigma}$ is the stress vector and $\mathbf{C}$ is the corresponding constitutive matrix. Once the crack appears, we assume that the bulk 
of the finite element $\Omega^{e} / \Gamma^{e}$ remains elastic and that the tractions at the crack line $\Gamma^{e}$ are related to the crack opening by some cohesive softening law. The crack appearance is governed by the failure criterion discussed further below. Since the governing fracture mode in brittle materials is mode $I$, the orientation of the crack $\boldsymbol{m}$ is chosen as perpendicular to the direction of the maximum tensile principal stress. The later is evaluated by using the average stress field in the element $\boldsymbol{\sigma}^{a v g}=\left[\sigma_{x}^{a v g}, \sigma_{y}^{a v g}, \sigma_{x y}^{a v g}\right]^{T}$.

We relate the tractions at a crack point $\boldsymbol{x} \in \Gamma^{e}$,

$$
\boldsymbol{t}=\left[t_{n}, t_{m}\right]^{T}
$$

to the crack opening $\overline{\overline{\boldsymbol{u}}}=\left[\overline{\bar{u}}_{n}, \overline{\bar{u}}_{m}\right]^{T}$ at that point, which is further defined in terms of the kinematic parameters $\alpha_{n 0}, \alpha_{n 1}, \alpha_{m 0}$ and $\alpha_{m 1}$. The chosen cohesive law that relates $\boldsymbol{t}$ and $\overline{\overline{\boldsymbol{u}}}$ is based on rigid-plasticity with softening. The failure criterion that checks for the initiation of the crack at $\boldsymbol{x} \in \Gamma^{e}$ and governs its further response is defined in terms of the tractions $\boldsymbol{t}$ and the stress-like softening variable $\overline{\bar{q}}$

$$
\overline{\bar{\phi}}=\overline{\bar{\phi}}(\boldsymbol{t}, \overline{\bar{q}}) \leq 0 \text {. }
$$

The later is defined in terms of the strain-like softening variable $\overline{\bar{\xi}}$, i.e. $\overline{\bar{q}}(\overline{\bar{\xi}})$. The remaining ingredients of the rigid-plasticity with softening are the evolution equations for the crack opening and the softening variable

$$
\dot{\overline{\boldsymbol{u}}}=\dot{\overline{\bar{\gamma}}} \frac{\partial \overline{\bar{\phi}}}{\partial \boldsymbol{t}}, \quad \dot{\overline{\bar{\xi}}}=\dot{\overline{\bar{\gamma}}} \frac{\partial \overline{\bar{\phi}}}{\partial \overline{\bar{q}}}
$$

the loading/unloading conditions, and the consistency condition

$$
\dot{\bar{\gamma}} \geq 0, \quad \overline{\bar{\phi}} \leq 0, \quad \dot{\bar{\gamma}} \overline{\bar{\phi}}=0, \quad \dot{\bar{\gamma}} \overline{\bar{\phi}}=0
$$

Here, $\dot{\bar{\gamma}} \geq 0$ is the plastic multiplier. The plastic dissipation that takes place at $\boldsymbol{x} \in \Gamma^{e}$ is given as $\overline{\bar{D}}^{p}=\boldsymbol{t}^{T} \dot{\overline{\boldsymbol{u}}}+\overline{\bar{q}} \overline{\overline{\bar{\xi}}}$.

\subsection{Equilibrium equations}

Let a two-dimensional body be discretized by the finite element mesh with $N_{e l}$ quadrilateral elements. The weak form of the equilibrium equations for a discretized body can be written as

$$
\mathbb{A}_{e=1}^{N_{e l}}\left(\delta \Pi^{i n t,(e)}-\delta \Pi^{e x t,(e)}\right)=0, \quad \delta \Pi^{i n t,(e)}=t^{(e)} \int_{\Omega^{e}} \hat{\boldsymbol{\epsilon}}^{T} \boldsymbol{\sigma} d \Omega
$$

Here, $\mathbb{A}$ is the assembly operator, $\delta \Pi^{i n t,(e)}$ and $\delta \Pi^{e x t,(e)}$ can be interpreted as virtual works of internal and external forces, respectively, $t^{(e)}$ is element's thickness and $\hat{\boldsymbol{\epsilon}}$ is vector of virtual strains. Interpolation of virtual strains is carried out in the same way as interpolation of real strains, see (11),

$$
\hat{\boldsymbol{\epsilon}}=\sum_{a=1}^{4} \boldsymbol{B}_{a} \hat{\boldsymbol{d}}_{a}+\hat{\boldsymbol{G}}_{n 0} \hat{\alpha}_{n 0}+\hat{\boldsymbol{G}}_{n 1} \hat{\alpha}_{n 1}+\hat{\boldsymbol{G}}_{m 0} \hat{\alpha}_{m 0}+\hat{\boldsymbol{G}}_{m 1} \hat{\alpha}_{m 1} .
$$


The crack opening parameters are viewed as element's additional degrees of freedom associated with the incompatible modes. Therefore, the interpolation matrices $\hat{\mathbf{G}}_{\text {mode }}$ are defined as

$$
\hat{\boldsymbol{G}}_{\text {mode }}=\boldsymbol{G}_{\text {mode }}-\frac{1}{A_{\Omega^{e}}} \int_{\Omega^{e}} \boldsymbol{G}_{\text {mode }} d \Omega, \quad \text { mode } \in[n 0, n 1, m 0, m 1],
$$

which ensures the convergence of the derived element in the spirit of the patch test (see e.g. [4]). By introducing (13)-(16) into (27), we obtain

$$
\begin{aligned}
& \hat{\boldsymbol{G}}_{n 0}=\underbrace{\overline{\boldsymbol{G}}_{n 0}-\frac{1}{A_{\Omega^{e}}} \int_{\Omega^{e}} \overline{\boldsymbol{G}}_{n 0} d \Omega-\frac{l_{\Gamma}}{A_{\Omega^{e}}} \boldsymbol{B}_{n} \boldsymbol{n}}_{\overline{\boldsymbol{G}}_{n 0}}+\delta_{\Gamma} \boldsymbol{B}_{n} \boldsymbol{n}, \\
& \hat{\boldsymbol{G}}_{n 1}=\underbrace{\overline{\boldsymbol{G}}_{n 1}-\frac{1}{A_{\Omega^{e}}} \int_{\Omega^{e}} \overline{\boldsymbol{G}}_{n 1} d \Omega}_{\widehat{\hat{\boldsymbol{G}}}_{n 1}}+\delta_{\Gamma} \boldsymbol{B}_{n} \boldsymbol{n} \xi_{\Gamma}, \\
& \hat{\boldsymbol{G}}_{m 0}=\underbrace{\overline{\boldsymbol{G}}_{m 0}-\frac{1}{A_{\Omega^{e}}} \int_{\Omega^{e}} \overline{\boldsymbol{G}}_{m 0} d \Omega-\frac{l_{\Gamma}}{A_{\Omega^{e}}} \boldsymbol{B}_{n} \boldsymbol{m}}_{\hat{\boldsymbol{G}}_{m 0}}+\delta_{\Gamma} \boldsymbol{B}_{n} \boldsymbol{m}, \\
& \hat{\boldsymbol{G}}_{m 1}=\underbrace{\overline{\boldsymbol{G}}_{m 1}-\frac{1}{A_{\Omega^{e}}} \int_{\Omega^{e}} \overline{\boldsymbol{G}}_{m 1} d \Omega}_{\overline{\hat{\boldsymbol{G}}}_{m 1}}+\delta_{\Gamma} \boldsymbol{B}_{n} \boldsymbol{m} \xi_{\Gamma} .
\end{aligned}
$$

A single element contribution to the internal virtual work can be now written as:

$$
\begin{aligned}
\delta \Pi^{i n t,(e)}= & \underbrace{\sum_{a=1}^{4} \hat{\boldsymbol{d}}_{a}^{T} \underbrace{t^{(e)} \int_{\Omega^{e}} \boldsymbol{B}_{a}^{T} \boldsymbol{\sigma} d \Omega}_{\boldsymbol{f}^{i n t,(e)}}}_{\text {standard }}+ \\
& \underbrace{t^{(e)} \int_{\Omega^{e}}\left(\hat{\alpha}_{n 0} \hat{\boldsymbol{G}}_{n 0}^{T}+\hat{\alpha}_{n 1} \hat{\boldsymbol{G}}_{n 1}^{T}+\hat{\alpha}_{m 0} \hat{\boldsymbol{G}}_{m 0}^{T}+\hat{\alpha}_{m 1} \hat{\boldsymbol{G}}_{m 1}^{T}\right) \boldsymbol{\sigma} d \Omega(32)}_{\text {additional }}
\end{aligned}
$$

From the term named "standard" in (32) the vector of element's internal nodal forces follows as

$$
\boldsymbol{f}^{i n t,(e)}=\left[\boldsymbol{f}_{a}^{i n t,(e)^{T}}\right]^{T}, \quad \boldsymbol{f}_{a}^{i n t,(e)}=t^{(e)} \int_{\Omega^{e}} \boldsymbol{B}_{a}^{T} \boldsymbol{\sigma} d \Omega .
$$

From $\delta \Pi^{e x t,(e)}$ the vector of element external nodal forces $\boldsymbol{f}^{e x t,(e)}$ follows as

$$
\delta \Pi^{e x t,(e)}=\sum_{a=1}^{4} \hat{\boldsymbol{d}}_{a}^{T} \boldsymbol{f}_{a}^{e x t,(e)}, \quad \boldsymbol{f}^{e x t,(e)}=\left[\boldsymbol{f}_{a}^{e x t,(e)^{T}}\right]^{T},
$$


representing the consistent external load applied to the element's nodes (the crack opening parameters do not contribute to the external load vector). The finite element mesh assembly of vectors $f^{i n t,(e)}$ and $f^{e x t,(e)}$ leads to a set of global equilibrium equations, see (25),

$$
\mathbb{A}_{e=1}^{N_{e l}}\left(f^{i n t,(e)}-f^{e x t,(e)}\right)=\mathbf{0}
$$

We have not used the term named "additional" in (32) when constructing the set of global equilibrium equations (34). We have rather chosen to express the contribution of that term to (25) on an element level. In view of (32) and (28)-(31), the following equations are obtained for each element of the mesh

$$
\begin{aligned}
& h_{n 0}^{(e)}=\underbrace{t^{(e)} \int_{\Omega^{e}} \overline{\hat{\boldsymbol{G}}}_{n 0}^{T} \boldsymbol{\sigma} d \Omega}_{h_{n 0}^{\Omega^{e}}}+\underbrace{t^{(e)} \int_{\Gamma^{e}} \underbrace{\boldsymbol{n}^{T} \boldsymbol{B}_{n}^{T} \boldsymbol{\sigma}}_{=t_{n}} d \Gamma}_{h_{n 0}^{\Gamma^{e}}}=0, \\
& h_{n 1}^{(e)}=\underbrace{t^{(e)} \int_{\Omega^{e}} \overline{\hat{\boldsymbol{G}}}_{n 1}^{T} \boldsymbol{\sigma} d \Omega}_{h_{n 1}^{\Omega^{e}}}+\underbrace{t^{(e)} \int_{\Gamma^{e}} \xi_{\Gamma} \underbrace{\boldsymbol{n}^{T} \boldsymbol{B}_{n}^{T} \boldsymbol{\sigma}}_{=t_{n}} d \Gamma}_{h_{n 1}^{\Gamma^{e}}}=0 \\
& h_{m 0}^{(e)}=\underbrace{t^{(e)} \int_{\Omega^{e}} \overline{\hat{\boldsymbol{G}}}_{m 0}^{T} \boldsymbol{\sigma} d \Omega}_{h_{m 0}^{\Omega^{e}}}+\underbrace{t^{(e)} \int_{\Gamma^{e}} \underbrace{\boldsymbol{m}^{T} \boldsymbol{B}_{n}^{T} \boldsymbol{\sigma}}_{=t_{m}} d \Gamma}_{h_{m 0}^{\Gamma^{e}}}=0 \\
& h_{m 1}^{(e)}=\underbrace{t^{(e)} \int_{\Omega^{e}} \overline{\hat{\boldsymbol{G}}}_{m 1}^{T} \boldsymbol{\sigma} d \Omega}_{h_{m 1}^{\Omega^{e}}}+\underbrace{t^{(e)} \int_{\Gamma^{e}} \xi_{\Gamma} \underbrace{\boldsymbol{m}^{T} \boldsymbol{B}_{n}^{T} \boldsymbol{\sigma}}_{=t_{m}} d \Gamma}_{h_{m 1}^{\Gamma^{e}}}=0, \quad \forall e \in\left[1, N_{e l}\right]
\end{aligned}
$$

Note, that $\int_{\Omega^{e}} \delta_{\Gamma}(\mathrm{o}) d \Omega=\int_{\Gamma^{e}}(\mathrm{o}) d \Gamma$ was used. The tractions $t_{n}$ and $t_{m}$ have been defined in (35)-(38). The $t_{n}$ represents the normal and the $t_{m}$ the tangential component of the traction $\mathbf{t}$ at a crack point. The vector form of equations (35)-(38) is:

$$
\boldsymbol{h}^{(e)}=\boldsymbol{h}^{\Omega^{e}}+\boldsymbol{h}^{\Gamma^{e}}=\left[h_{n 0}^{\Omega^{e}}, h_{n 1}^{\Omega^{e}}, h_{m 0}^{\Omega^{e}}, h_{m 1}^{\Omega^{e}}\right]^{T}+\left[h_{n 0}^{\Gamma^{e}}, h_{n 1}^{\Gamma^{e}}, h_{m 0}^{\Gamma^{e}}, h_{m 1}^{\Gamma^{e}}\right]^{T}=\mathbf{0}
$$

A Gauss integration scheme with $2 \times 2$ points is used for the numerical integration of the integrals of the form $\int_{\Omega^{e}} g(x, y) d \Omega$. A 2-point Gauss integration scheme is used for the numerical integration of the integrals of the form $\int_{\Gamma^{e}} f\left(\xi_{\Gamma}\right) d \Gamma$, see Figure 2. The integration along the crack is thus carried out as

$$
\int_{-\frac{l_{\Gamma}}{2}}^{\frac{l_{\Gamma}}{2}} f\left(\xi_{\Gamma}\right) d \xi=\sum_{c i p=1}^{2} f\left(\xi_{\Gamma}^{c i p}\right) w^{c i p} \frac{l_{\Gamma}}{2}, \xi_{\Gamma}^{c i p}= \pm \frac{l_{\Gamma}}{2 \sqrt{3}}, w^{c i p}=1
$$


where $f\left(\xi_{\Gamma}\right)$ in a scalar function, $f\left(\xi_{\Gamma}^{c i p}\right)$ is its value at the Gauss point with coordinate $\xi_{\Gamma}^{c i p}$ and $w^{c i p}$ is its corresponding weight. By using (40), the $\boldsymbol{h}^{\Gamma^{e}}$ of equation (39) can be written as

$$
\boldsymbol{h}^{\Gamma^{e}}=\frac{t^{(e)} l_{\Gamma}}{2}\left[t_{n}^{1}+t_{n}^{2}, \xi_{\Gamma}^{1} t_{n}^{1}+\xi_{\Gamma}^{2} t_{n}^{2}, t_{m}^{1}+t_{m}^{2}, \xi_{\Gamma}^{1} t_{m}^{1}+\xi_{\Gamma}^{2} t_{m}^{2}\right]^{T}
$$

where $\boldsymbol{t}^{1}=\left[t_{n}^{1} t_{m}^{1}\right]^{T}$ is the traction in the first integration point and $\boldsymbol{t}^{2}=$ $\left[\begin{array}{ll}t_{n}^{2} & t_{m}^{2}\end{array}\right]^{T}$ is the traction in the second integration point.

In order to get solution (the displacements at nodes of the finite element mesh and the crack-opening parameters of each finite element), equations (34) and (39) need to be solved.

\subsection{Solution procedure}

The solution of the set of global nonlinear equations in (34) and of the set of local nonlinear equations in (39) is obtained at discrete pseudo-time values $0, \tau_{1}, \tau_{2}, \ldots, \tau_{n-1}, \tau_{n}, \tau_{n+1}, \ldots, T$ by means of the incremental-iterative scheme. We will consider a typical pseudo-time increment from $\tau_{n}$ to $\tau_{n+1}$. Let us assume that all necessary variables at $\tau_{n}$, i.e. $\boldsymbol{d}_{n}^{(e)}=\left[\boldsymbol{d}_{1, n}^{T}, \boldsymbol{d}_{2, n}^{T}, \boldsymbol{d}_{3, n}^{T}, \boldsymbol{d}_{4, n}^{T}\right]^{T}, \boldsymbol{\alpha}_{n}^{(e)}=$ $\left[\alpha_{n 0, n}, \alpha_{n 1, n}, \alpha_{m 0, n}, \alpha_{m 1, n}\right]^{T}$ and $\overline{\bar{\xi}}_{n}^{c i p}$ are given for each element $(e)$. The iterations in the pseudo-time step (with $(i)$ as the iteration counter) are then performed in order to compute the converged values of those variables at $\tau_{n+1}$, i.e. $\boldsymbol{d}_{n+1}^{(e)}, \boldsymbol{\alpha}_{n+1}^{(e)}$ and $\overline{\bar{\xi}}_{n+1}^{c i p}$. This computation is split into two phases:

(a) The global phase computes the current iterative values of nodal displacements at $\tau_{n+1}$ as $\boldsymbol{d}_{n+1}^{(e),(i)}=\boldsymbol{d}_{n+1}^{(e),(i-1)}+\Delta \boldsymbol{d}_{n+1}^{(e),(i-1)}$, while keeping the other variables fixed. The computation of iterative update $\Delta \boldsymbol{d}_{n+1}^{(e),(i-1)}$ will be explained further below.

(b) The local phase computes the new values of crack variables $\boldsymbol{\alpha}_{n+1}^{(e)}, \overline{\bar{\xi}}_{n+1}^{c i p}$ while keeping $\boldsymbol{d}_{n+1}^{(e),(i)}$ fixed. This procedure is activated if the considered element has a crack or it is at the crack front.

Let us first describe the phase (b). It is carried out only in those finite elements where: (i) the element had a crack at the end of the previous pseudotime step $\tau_{n}$; or (ii) the element had no crack at the end of the previous pseudotime step but there was a crack in one of the neighboring elements. In the latter case we first compute, by using the average stress field, the direction of the possible crack $\mathbf{m}$, the normal to the possible crack $\mathbf{n}$, and related geometry $\left(x_{\Gamma E}, \Omega^{e-}, \Omega^{e+}, l_{\Gamma}\right)$.

The computation of crack variables starts with the computation of trial values for tractions in crack integration points. Equilibrium equations (39) are 
used. With $\boldsymbol{h}_{n+1}^{\Omega^{e}, \text { trial }}=\boldsymbol{h}^{\Omega^{e}}\left(\boldsymbol{\sigma}\left(\boldsymbol{d}_{n+1}^{(e),(i)}, \boldsymbol{\alpha}_{n}\right)\right)$ and (41) a system of four algebraic equations

$$
\boldsymbol{h}_{n+1}^{\Omega^{e}, \text { trial }}+\boldsymbol{h}^{\Gamma^{e}}\left(\boldsymbol{t}_{n+1}^{1, \text { trial }}, \boldsymbol{t}_{n+1}^{2, \text { trial }}\right)=\mathbf{0},
$$

is obtained that provides two trial tractions at each crack integration point, i.e. $\boldsymbol{t}_{n+1}^{1, \text { trial }}=\left[t_{n, n+1}^{1, \text { trial }}, t_{m, n+1}^{1, \text { trial }}\right]^{T}, \boldsymbol{t}_{n+1}^{2, \text { trial }}=\left[t_{n, n+1}^{2, \text { trial }}, t_{m, n+1}^{2, \text { trial }}\right]^{T}$. The trial failure criteria is further evaluated

$$
\underbrace{\left.\overline{\bar{\phi}}^{1, \text { trial }}=\overline{\bar{\phi}}\left(\boldsymbol{t}_{n+1}^{1, \text { trial }}, \overline{\bar{q}}\left(\overline{\bar{\xi}}_{n}^{1}\right)\right) \leq 0 \text { or } \overline{\bar{\phi}}^{2, \text { trial }}=\overline{\bar{\phi}}\left(\boldsymbol{t}_{n+1}^{2, \text { trial }}, \overline{\bar{q}}_{\overline{\bar{\xi}}} \overline{\bar{\xi}}_{n}^{2}\right)\right) \leq 0}_{?} .
$$

If the criterion (43) is satisfied, the values of softening plasticity local variables remain unchanged

$$
\overline{\bar{\phi}}^{1, \text { trial }} \leq 0 \text { or } \overline{\bar{\phi}}^{2, \text { trial }} \leq 0 \Longrightarrow \boldsymbol{\alpha}_{n+1}^{(e)}=\boldsymbol{\alpha}_{n}^{(e)}, \quad \overline{\bar{\xi}}_{n+1}^{1}=\overline{\bar{\xi}}_{n}^{1}, \overline{\bar{\xi}}_{n+1}^{2}=\overline{\bar{\xi}}_{n}^{2} .
$$

It means that that the crack has not appeared in the case of (ii) above. It also means that the opening of the crack has not changed in the case of (i) above. If (43) is violated, the values of crack openings are updated by backward Euler integration scheme

$$
\begin{array}{ll}
\overline{\overline{\boldsymbol{u}}}_{n+1}^{1}=\overline{\overline{\boldsymbol{u}}}_{n}^{1}+\left.\overline{\bar{\gamma}}_{n+1}^{1} \frac{\partial \overline{\bar{\phi}}}{\partial \boldsymbol{t}}\right|_{\xi_{\Gamma}^{1}}, & \overline{\bar{\xi}}_{n+1}^{1}=\overline{\bar{\xi}}_{n}^{1}+\left.\overline{\bar{\gamma}}_{n+1}^{1} \frac{\partial \overline{\bar{\phi}}}{\partial q}\right|_{\xi_{\Gamma}^{1}}, \\
\overline{\overline{\boldsymbol{u}}}_{n+1}^{2}=\overline{\overline{\boldsymbol{u}}}_{n}^{2}+\left.\overline{\bar{\gamma}}_{n+1}^{2} \frac{\partial \overline{\bar{\phi}}}{\partial \boldsymbol{t}}\right|_{\xi_{\Gamma}^{2}}, & \overline{\bar{\xi}}_{n+1}^{2}=\overline{\bar{\xi}}_{n}^{2}+\left.\overline{\bar{\gamma}}_{n+1}^{2} \frac{\partial \overline{\bar{\phi}}}{\partial q}\right|_{\xi_{\Gamma}^{2}},
\end{array}
$$

where $\overline{\bar{\gamma}}_{n+1}^{1}=\dot{\bar{\gamma}}_{n+1}^{1}\left(\tau_{n+1}-\tau_{n}\right)$ and $\overline{\bar{\gamma}}_{n+1}^{2}=\dot{\bar{\gamma}}_{n+1}^{2}\left(\tau_{n+1}-\tau_{n}\right)$. The values of $\overline{\bar{\gamma}}_{n+1}^{1}$ and $\overline{\bar{\gamma}}_{n+1}^{2}$ are obtained by solving the system of two nonlinear equations

$$
\begin{aligned}
& \overline{\bar{\phi}}^{1}\left(\boldsymbol{t}_{n+1}^{1}\left(\boldsymbol{\alpha}_{n+1}^{(e)}\left(\overline{\bar{\gamma}}_{n+1}^{1}, \overline{\bar{\gamma}}_{n+1}^{2}\right)\right), \overline{\bar{q}}\left(\overline{\bar{\xi}}_{n+1}^{1}\left(\overline{\bar{\gamma}}_{n+1}^{1}\right)\right)\right)=\overline{\bar{\phi}}^{1}\left(\overline{\bar{\gamma}}_{n+1}^{1}, \overline{\bar{\gamma}}_{n+1}^{2}\right)=0, \\
& \overline{\bar{\phi}}^{2}\left(\boldsymbol{t}_{n+1}^{2}\left(\boldsymbol{\alpha}_{n+1}^{(e)}\left(\overline{\bar{\gamma}}_{n+1}^{1}, \overline{\bar{\gamma}}_{n+1}^{2}\right)\right), \overline{\bar{q}}^{2}\left(\overline{\bar{\xi}}_{n+1}^{2}\left(\overline{\bar{\gamma}}_{n+1}^{2}\right)\right)\right)=\overline{\bar{\phi}}^{2}\left(\overline{\bar{\gamma}}_{n+1}^{1}, \overline{\bar{\gamma}}_{n+1}^{2}\right)=0 .
\end{aligned}
$$

In (47)-(48) the relations between the jumps in displacements at the integration points $\overline{\overline{\boldsymbol{u}}}^{1}, \overline{\overline{\boldsymbol{u}}}^{2}$ and the crack-opening kinematic parameters $\boldsymbol{\alpha}^{(e)}=\boldsymbol{\alpha}^{(e)}\left(\overline{\overline{\boldsymbol{u}}}^{1}, \overline{\overline{\boldsymbol{u}}}^{2}\right)$ are needed, which are easy to get. We can thus determine the updated values of $\boldsymbol{\alpha}_{n+1}^{(e)}=\boldsymbol{\alpha}^{(e)}\left(\overline{\overline{\boldsymbol{u}}}_{n+1}^{1}\left(\overline{\bar{\gamma}}_{n+1}^{1}\right), \overline{\overline{\boldsymbol{u}}}_{n+1}^{2}\left(\overline{\bar{\gamma}}_{n+1}^{2}\right)\right)$ once the values of the plastic multipliers $\overline{\bar{\gamma}}_{n+1}^{1}$ and $\overline{\bar{\gamma}}_{n+1}^{2}$ are known. The final result of the above described softening plasticity procedure are the new values of parameters $\boldsymbol{\alpha}_{n+1}^{(e)}$, which influence the stress state of the element by giving the new values of stress in the bulk integration points bip as

$$
\boldsymbol{\sigma}_{n+1}^{b i p}=\boldsymbol{C} \overline{\boldsymbol{\epsilon}}\left(\boldsymbol{d}_{n+1}^{(e),(i)}, \boldsymbol{\alpha}_{n+1}^{(e)}\right) .
$$


Once the local variables are computed, we turn to the global phase (a). We perform a new iterative loop, if needed, in order to provide new iterative values of nodal displacements. First, the set of global equilibrium equations (34) is checked with newly computed $\boldsymbol{\sigma}_{n+1}^{b i p}$ from the phase (b)

$$
\left\|\mathbb{A}_{e=1}^{N_{e l}}\left[f_{n+1}^{i n t,(e)}-f_{n+1}^{e x t,(e),(i)}\right]\right\| \stackrel{?}{<} \text { tol. }
$$

If the convergence criterion (50) is satisfied, we move on to the next pseudo-time incremental step. If the convergence criterion fails, we perform a new iterative sweep within the present pseudo-time incremental step. New iterative values of nodal generalized displacements of the finite element mesh are computed by accounting for each element contribution. A single element contribution can be written as

$$
\left[\begin{array}{ll}
\boldsymbol{K}^{(e)} & \boldsymbol{K}^{f \alpha} \\
\boldsymbol{K}^{h d} & \boldsymbol{K}^{h \alpha}
\end{array}\right]_{n+1}^{(i)}\left(\begin{array}{c}
\Delta \boldsymbol{d}_{n+1}^{(e),(i)} \\
\Delta \boldsymbol{\alpha}_{n+1}^{(e),(i)}
\end{array}\right)=\left(\begin{array}{c}
\boldsymbol{f}_{n+1}^{e x t,(e)}-\boldsymbol{f}_{n+1}^{i n t,(e),(i)} \\
\mathbf{0}
\end{array}\right),
$$

where the parts of the element stiffness matrix can be formally written as

$$
\begin{array}{cc}
\boldsymbol{K}_{n+1}^{(e),(i)}=\left(\frac{\partial \boldsymbol{f}^{i n t,(e)}}{\partial \boldsymbol{d}^{(e)}}\right)_{n+1}^{(i)}, & \boldsymbol{K}_{n+1}^{f \alpha,(i)}=\left(\frac{\partial \boldsymbol{f}^{i n t,(e)}}{\partial \boldsymbol{\alpha}^{(e)}}\right)_{n+1}^{(i)}, \\
\boldsymbol{K}_{n+1}^{h d,(i)}=\left(\frac{\partial \boldsymbol{h}^{(e)}}{\partial \boldsymbol{d}^{(e)}}\right)_{n+1}^{(i)}, & \boldsymbol{K}_{n+1}^{h \alpha,(i)}=\left(\frac{\partial \boldsymbol{h}^{(e)}}{\partial \boldsymbol{\alpha}^{(e)}}\right)_{n+1}^{(i)}
\end{array}
$$

The static condensation in (51) allows to get the standard form of the element stiffness matrix $\widehat{\boldsymbol{K}}_{n+1}^{(e),(i)}$ that contributes to the finite element assembly

$$
\mathbb{A}_{e=1}^{N_{e l}}\left(\widehat{\boldsymbol{K}}_{n+1}^{(e),(i)} \Delta \boldsymbol{d}_{n+1}^{(i)}\right)=\mathbb{A}_{e=1}^{N_{e l}}\left(\boldsymbol{f}_{n+1}^{e x t,(e)}-\boldsymbol{f}_{n+1}^{i n t,(e),(i)}\right),
$$

where

$$
\widehat{\mathbf{K}}_{n+1}^{(e),(i)}=\boldsymbol{K}_{n+1}^{(e),(i)}-\boldsymbol{K}_{n+1}^{f \alpha,(i)}\left(\boldsymbol{K}_{n+1}^{h \alpha,(i)}\right)^{-1} \boldsymbol{K}_{n+1}^{h d,(i)} .
$$

Solution of (53) gives the values of iterative update $\Delta \boldsymbol{d}_{n+1}^{(e),(i)}$.

\section{$3 \quad$ Examples}

In this section we provide the results of numerical simulations of two well known tests to illustrate a very satisfying performance of the proposed finite element. The finite element code was generated by using symbolic manipulation code AceGen and the examples were computed by using finite element program AceFem, both developed by Korelc, see [8]. 


\subsection{Three point bending test}

We consider a classical benchmark problem of a notched concrete beam subjected to three point loads, see [5] and references therein. Similar test was considered by other authors, e.g. [14]. In Figure 3 we present the geometry of the specimen, a $200 \mathrm{~cm} \times 20 \mathrm{~cm} \times 5 \mathrm{~cm}$ simply supported concrete beam with a $2 \mathrm{~cm} \times 10 \mathrm{~cm} \times 5 \mathrm{~cm}$ notch placed at the bottom center of the beam. The beam is loaded by a downward displacement imposed at its top center. The material characteristics of the material are: Young's modulus $E=3000 \mathrm{kN} / \mathrm{cm}^{2}$, Poisson's ratio $\nu=0.2$ and the ultimate tensile strength $\sigma_{u}=3.33 \mathrm{kN} / \mathrm{cm}^{2}$. The crack propagates from the notch in mode $I$ fashion, therefore we define the cohesive response in the crack only in its normal direction. The crack response in its tangential direction is not considered in simulations. The tensile failure criterion (55) is of the form

$$
\overline{\bar{\phi}}(\boldsymbol{t}, \overline{\bar{q}})=t_{n}-\left(\sigma_{u}-\overline{\bar{q}}\right) \leq 0 .
$$

and the softening curve is

$$
\overline{\bar{q}}=\sigma_{u}\left(1-\exp ^{-\frac{\overline{\bar{\xi}} \sigma_{u}}{\bar{G}_{f}}}\right),
$$

where $G_{f}=0.124 \cdot 10^{-2} \mathrm{kN} / \mathrm{cm}$ is the fracture energy. Note, that $\overline{\bar{\xi}}=\overline{\bar{u}}_{n}$. The assumed rigid-plastic softening curve in the normal direction is presented in Figure 4.

In Figure 5 we present two finite element meshes that were used. The coarser mesh consists of 530 finite elements and the finer one of 2186 finite elements. On the left side of Figure 6 we plot the reaction force $R_{y}$ versus the imposed displacement $u_{y}$ for both meshes. The crack starts at the notch when $t_{n}$ reaches the tensile strength $\sigma_{u}$. It has been chosen that the crack propagates in the direction perpendicular to the maximum principal stress, i.e. in the mode $I$ fashion. However, a problem was encountered when using the above criterion to determine the crack direction. Namely, the direction of the maximum principal stress at some point suddenly changes for 90 degrees. This causes a problem in convergence with the fine mesh and a non-physical response with the coarse mesh, see left side of Figure 6. The crack direction problem was also reported in [2], [10] and [13] and we direct the reader therein for further discussion. One of the possible solutions is to define crack propagation in the direction of the maximum circumferential stress that is evaluated on a circle of a small diameter centered at the crack tip.

In order to obtain a solution we predetermined direction of the crack. The crack was allowed to propagate perpendicular to the length of the beam, which is expected for mode $I$ situation. With this modification we obtained results that are within the experimentally established bounds (the experimental results have been taken from [5]). The results of all simulations are given in Figure 6 (left). In the center and right side of Figure 6 we present the deformed configuration (magnified 100 times) of the area near the notch. 


\subsection{Four point bending test}

We consider another classical benchmark problem - the four point bending test of a concrete beam with a notch, see [5] and references therein. In Figure 7 we present the specimen's geometry together with loading and supports. The material characteristics are: Young's modulus $E=2880 \mathrm{kN} / \mathrm{cm}^{2}$, Poisson's ratio $\nu=0.18$ and the ultimate tensile strength $\sigma_{u}=2.8 \mathrm{kN} / \mathrm{cm}^{2}$. The initiation and behavior of the crack is governed by the failure criterion (55) and the softening curve

$$
\overline{\bar{q}}=\max \left[\sigma_{u},-K_{s} \overline{\bar{\xi}}\right],
$$

with softening modulus $K_{s}=-39.2 \mathrm{kN} / \mathrm{cm}^{2}$. In Figure 8 we present the mesh used in simulations. With respect to the description of the crack opening we considered three cases: (i) " $n 0+m 0 "$ - constant jump in displacements in both normal and tangential direction, (ii) " $n 0+n 1 "$ - linear jump in displacement in normal direction only and (iii) " $n 0 "$ - constant jump in displacements in normal direction only. In the case " $n 0+m 0$ " we considered a reduced shear stiffness for the tangential response according to

$$
\overline{\bar{\phi}}\left(t_{m}\right)=t_{m}-k_{m} \overline{\bar{u}}_{m}=0,
$$

where $k_{m}=2.88 \mathrm{kN} / \mathrm{cm}^{3}$.

The results are presented in Figures 9 and 10. On the left side of Figure 9 we plot the applied load versus crack mouth sliding displacement. The results are compared with those from [5]. We can see that all used formulations give good prediction of the limit load, while only the mixed mode formulation " $n 0+m 0$ " was able to capture the softening response. On the right side of Figure 9 we plot the crack paths that correspond to curves on the left side of the same figure. In Figure 10 we present the deformed (magnified 200 times) mesh of the area near the notch. The crack paths presented in Figure 9 and the deformed meshes presented in Figure 10 are in agreement with those from [5].

\section{Conclusions}

A finite element with embedded strong discontinuity has been presented and used to model the fracture process in two-dimensional concrete solids. The element has linear interpolations of the displacements jumps (in both normal and tangential directions), which are important for its locking-free response. The used cohesive law in the crack, which is based on rigid-plasticity with softening, has proven to be a reasonable model for representation of the crack behavior, when the loading is increasing monotonically. With this constitutive model, the simulations of two well known benchmark tests have provided very satisfying results. In order to make the crack growth algorithm more robust, the continuity of the crack has been enforced. As shown by the first numerical example, the tracking of the direction of the crack propagation may be false, when related only to the local stress state in a single element. 


\section{References}

\section{References}

[1] J. Dujc, B. Brank, A. Ibrahimbegovic, Quadrilateral finite element with embedded strong discontinuity for failure modeling in solids, submitted.

[2] J. Mosler, On advanced solution strategies to overcome locking effects in strong discontinuity approaches, Int. J. Numer. Meth. Engng., 63, 13131341, 2005.

[3] A. Ibrahimbegovic, Nonlinear Solid Mechanics: Theoretical Formulations and Finite Element Solution Methods, Springer, 2009.

[4] A. Ibrahimbegovic, E.L. Wilson, A modified method of incompatible modes. Commun. Appl. Numer. Meth., 7, 187-194, 1991.

[5] C. Linder, F. Armero, Finite elements with embedded strong discontinuities for the modeling of failure in solids, Int. J. Numer. Meth. Engng., 72, 13911433, 2007.

[6] A. Ibrahimbegovic, D. Brancherie, Combined hardening and softening constitutive model of plasticity: precursor to shear slip line failure, Comput. Mech., 31, 88-100, 2003.

[7] J. Dujc, B. Brank, On stress resultant plasticity and viscoplasticity for metal plates, Finite Elements in Analysis and Design, 44, 174-185, 2008.

[8] J. Korelc, AceGen, AceFem, available at http://www.fgg.uni-lj.si/Symech.

[9] Manzoli O.L., Shing P.B., A general technique to embed non-uniform discontinuities into standard solid finite elements, Computers and Structures, 84, 742-757, 2006.

[10] J.M. Sancho, J. Planas, D.A. Cendón, E. Reyes, J.C. Gálvez, An embedded crack model for finite element analysis of concrete structure, Engineering Fracture Mechanics, 74, 75-86, 2007.

[11] M. Jirásek, T. Zimmermann, Embedded crack model: I. Basic formulation, International journal for numerical methods in engineering, 50, 1269-1290, 2001.

[12] D. Brancherie, A. Ibrahimbegovic, Novel anisotropic continuum-discrete damage model capable of representing localized failure of massive structures. Part I: theoretical formulation and numerical implementation, Engineering Computations, 26, 100-127, 2009.

[13] M. Jirásek, T. Zimmermann, Embedded crack model. Part II: Combination with smeared cracks, International journal for numerical methods in engineering, 50, 1291-1305, 2001. 
[14] J.L. Asferg, P.N. Poulsen, L.O. Nielsen, A direct XFEM formulation for modeling of cohesive crack growth in concrete, Computers and Concrete, 4, 83-100, 2007.

[15] O.L. Manzoli, J. Oliver, A.E. Huespe, G. Diaz, A mixture theory based method for three-dimensional modeling of reinforced concrete members with embedded crack finite elements, Computers and Concrete, 5, 401-416, 2008.

[16] C. Feist, G. Hofstetter, Validation of 3D crack propagation in plain concrete. Part I: Experimental investigation - the PCT3D test, Computers and Concrete, 4, 49-66, 2007.

[17] T.C. Gasser, Validation of 3D crack propagation in plain concrete. Part II: Computational modeling and predictions of the PCT3D test, Computers and Concrete, 4, 67-82, 2007.

[18] P. Jehel, L. Davenne, A. Ibrahimbegovic, P. Léger, Towards robust viscoelastic-plastic-damage material model with different hardenings/softenings capable of representing salient phenomena in seismic loading applications, Computers and Concrete (in print).

[19] N. Dominguez, M.A. Fernández, Enhanced solid element for modeling of reinforced concrete structures with bond-slip, Computers and Concrete (in print). 

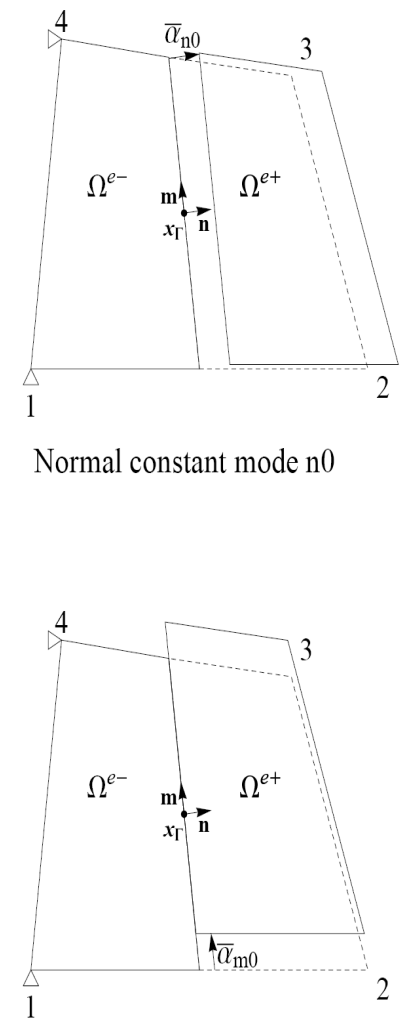

Tangential constant mode $\mathrm{m} 0$
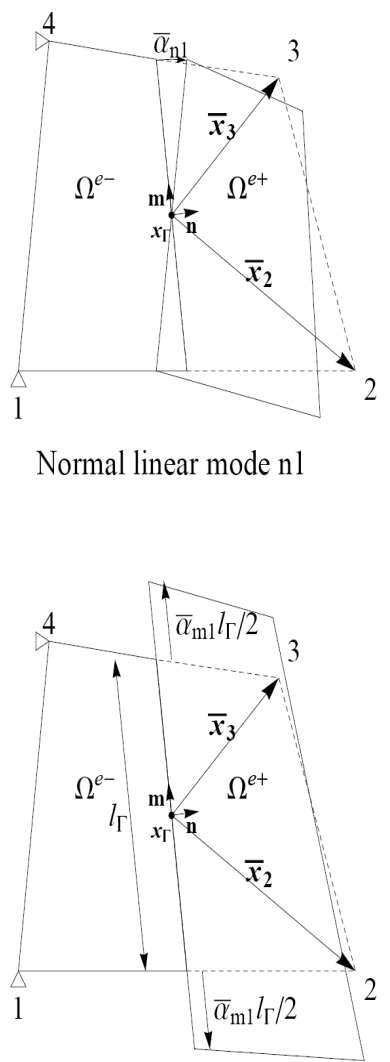

Tangential linear mode $\mathrm{ml}$

Figure 1: Different crack opening modes of quadrilateral finite element with embedded crack. 


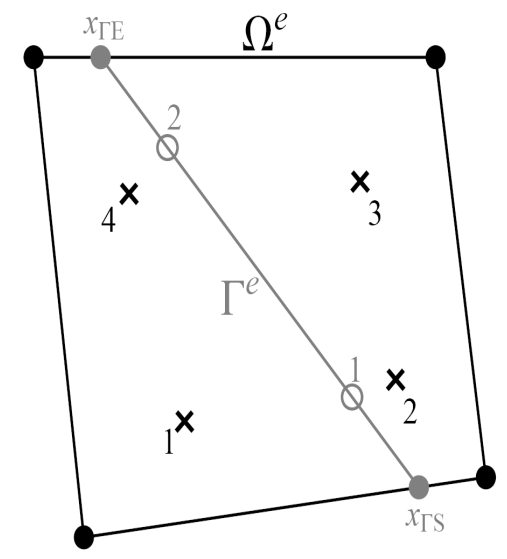

Figure 2: Integration points of the quadrilateral finite element with embedded crack

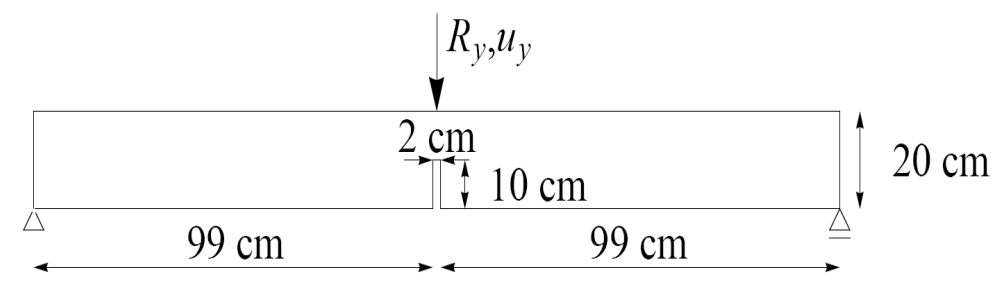

Figure 3: Three point bending test: geometry data.

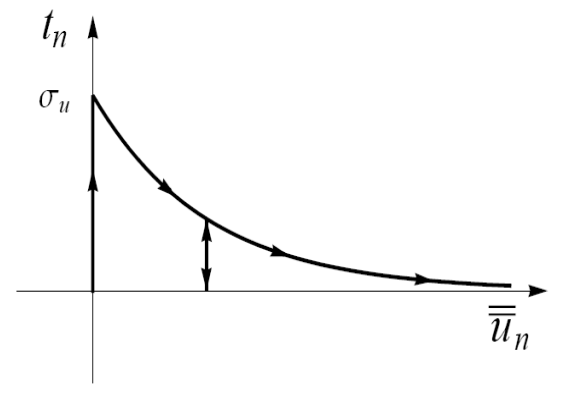

Figure 4: Rigid-plastic cohesive law in crack normal direction. 

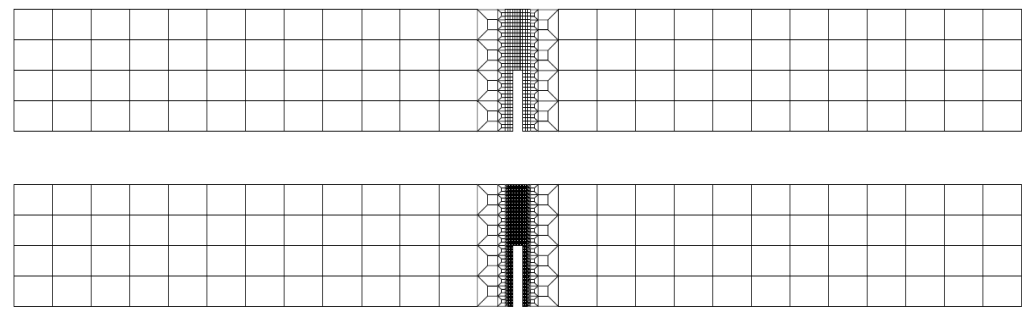

Figure 5: Coarse and fine meshes for the three-point bending test.
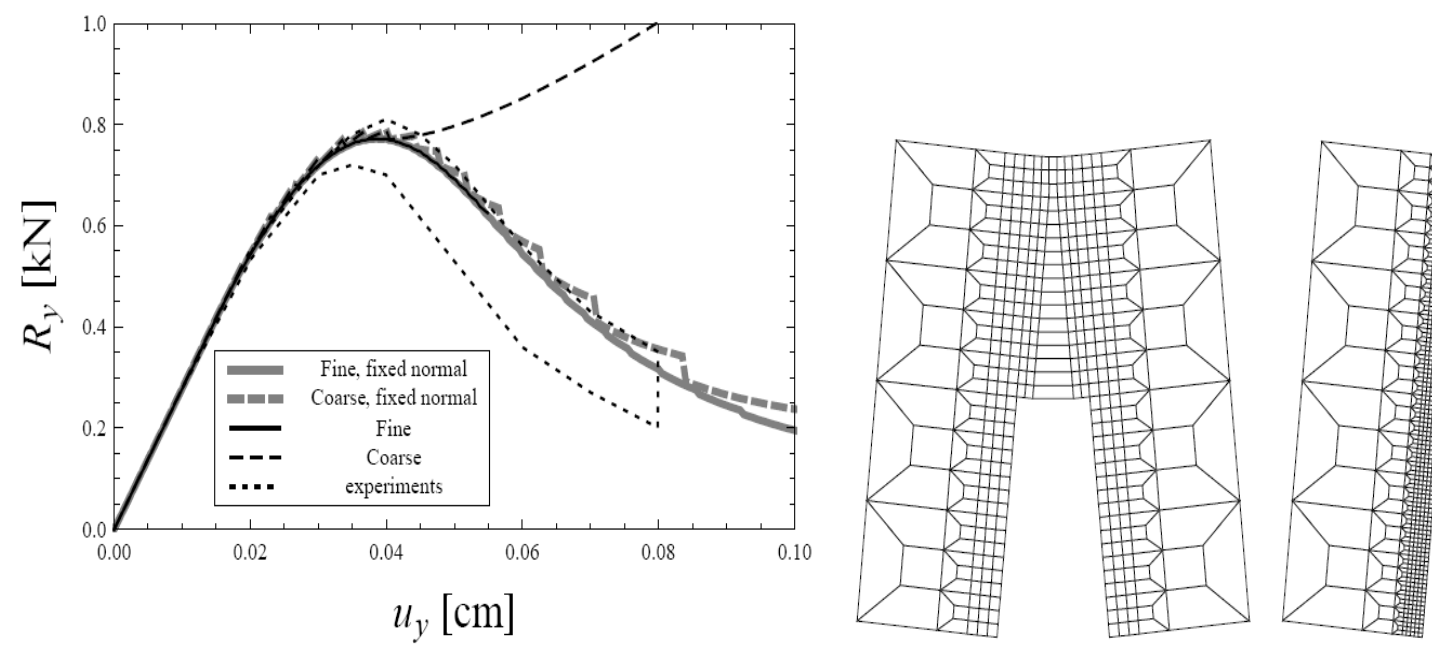

Figure 6: Force versus imposed displacement curves (left). Deformed meshes around the notch (right). 


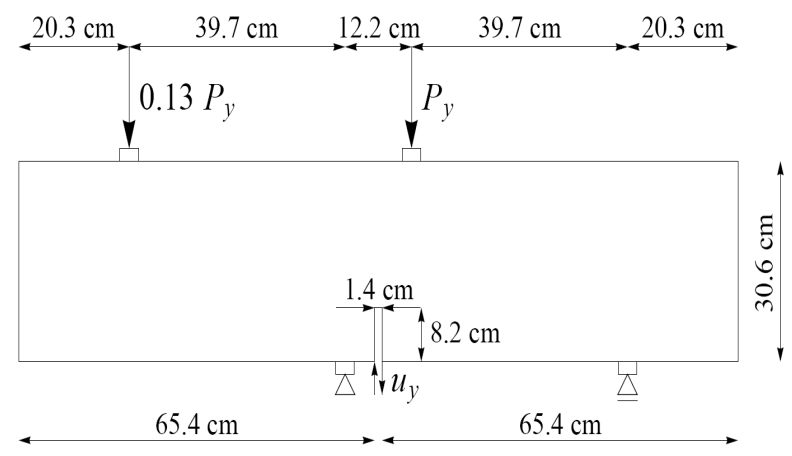

Figure 7: Four point bending test: geometry and loading.

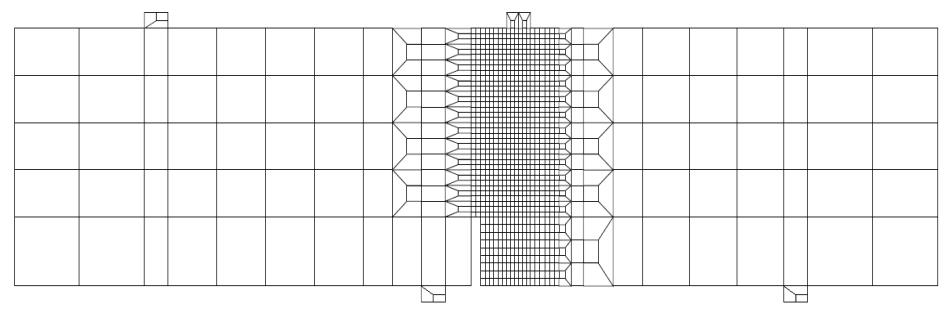

Figure 8: Four point bending test: geometry and loading. 

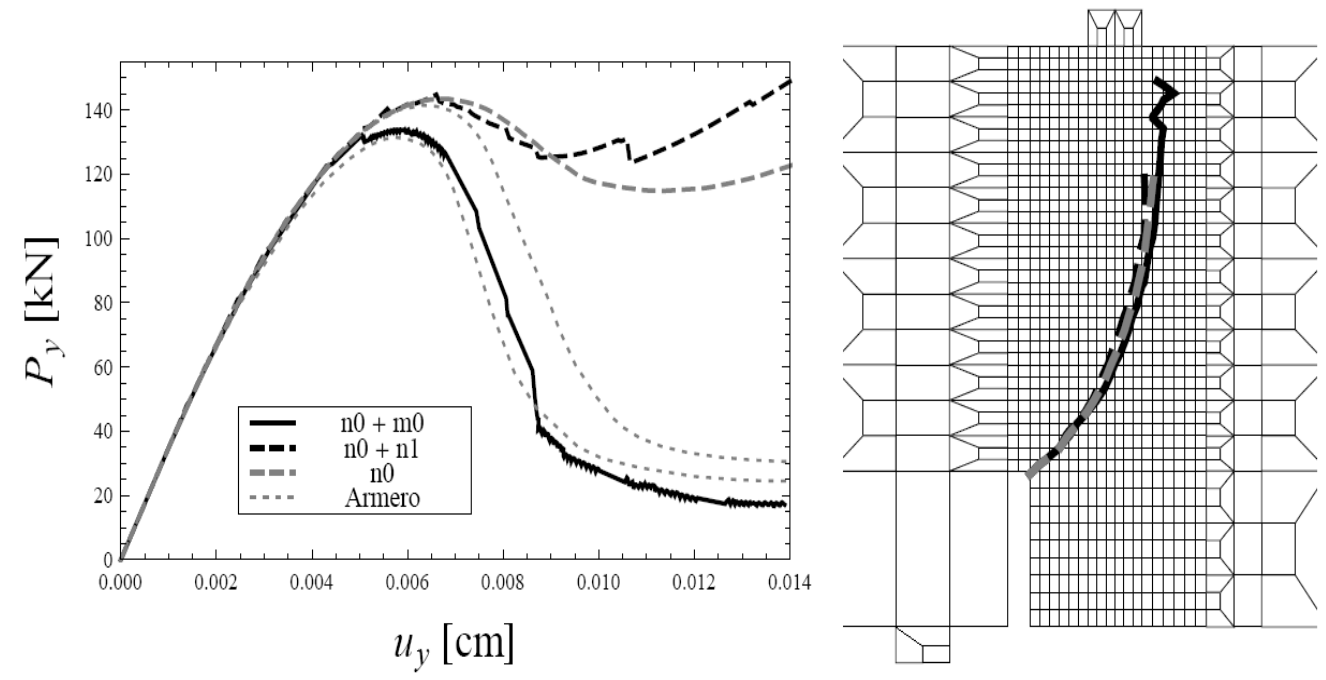

Figure 9: Load versus crack mouth sliding displacement curves (left). The corresponding crack paths (right).

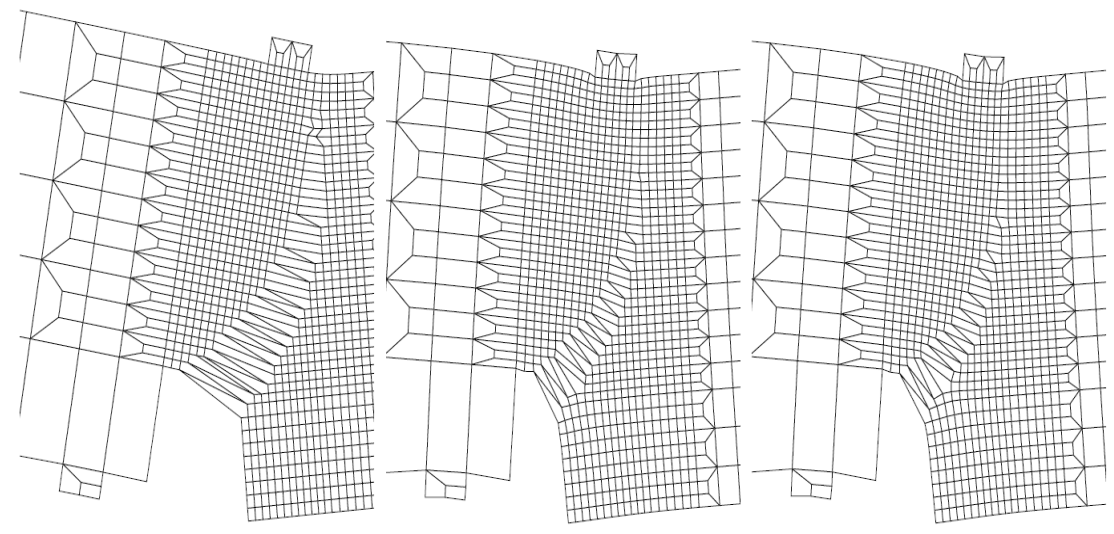

Figure 10: Deformed meshes for " $n 0+m 0 ", " n 0+n 1 "$ and " $n 0$ " formulations. 